\title{
Grand Challenges for Wireless Sensor Networks
}

\author{
Ian F. Akyildiz \\ Broadband and Wireless Networking Lab, \\ School of Electrical and Computer Engineering, \\ Georgia Institute of Technology, \\ Atlanta, GA 30332 \\ USA \\ ian@ece.gatech.edu
}

\begin{abstract}
The technological advances in the micro-electro-mechanical systems (MEMS) and the wireless communications have enabled the deployment of the small intelligent sensor nodes at homes, in workplaces, supermarkets, plantations, oceans, streets, and highways to monitor the environment. The realization of smart environments to improve the efficiency of nearly every aspect of our daily lives by enhancing the humanto-physical world interaction is one of the most exciting potential sensor network applications utilizing these intelligent sensor nodes. However, this objective necessitates the efficient and application specific communication protocols to assure the reliable communication of the sensed event features and hence enable the required actions to be taken by the actors in the smart environment. In this talk, the grand challenges for the design and development of sensor/actor network communication protocols are presented. More specifically, application layer, transport layer, network layer, data link layer, in particular, error control and MAC protocols, and physical layer as well as cross layer issues are explained in detail.
\end{abstract}

